\title{
DOENÇA PERIODONTAL COMO POSSÍVEL FATOR DE RISCO PARA ATEROSCLEROSE CORONARIANA
}

\section{PERIODONTAL DISEASE AS A POSSIBLE FACTOR OF RISK FOR CORONARY ATHEROSCLEROSIS}

\author{
Chigueyuki Jitumori ${ }^{1,3}$, Marcelo Ferraz de Freitas ${ }^{3}$, Eduardo Pietruchinski ${ }^{3}$, \\ Jeanine Isabel Margraf Bittencourt ${ }^{2,3}$, Josiane Padilha de Paula ${ }^{2,3}$, \\ Marcelo Valladão ${ }^{3}$, Vitoldo Antonio Kozlowski Junior². \\ Recebido para publicação em 27/05/2010 \\ Aceito para publicação em 24/06/2010 \\ ${ }^{1}$ Autor para contato: Mestrado em Clínica Odontológica/Universidade Estadual de \\ Ponta Grossa-PR. E-mail: cjitumori@gmail.com; (42)3222-3614/3235-2443/9905-3940 \\ ${ }^{2}$ Universidade Estadual de Ponta Grossa - PR \\ ${ }^{3}$ Hospital Bom Jesus - Ponta Grossa - PR
}

\section{RESUMO}

Diversos trabalhos científicos mostraram uma associação significativa entre parâmetros periodontais e problemas cardiovasculares. O objetivo desta pesquisa foi avaliar a saúde bucal e a doença periodontal em humanos verificando se essas condições poderiam estar correlacionadas como possíveis fatores de risco para doença aterosclerótica coronariana. Oitenta pacientes foram submetidos à cinecoronariografia e divididos em dois grupos: indivíduos com doença aterosclerótica coronariana $(\mathrm{n}=59)$ e indivíduos sem doença aterosclerótica coronariana $(\mathrm{n}=21)$. As variáveis analisadas foram idade, sexo, tabagismo, etilismo, hipertensão arterial sistêmica, diabete melito, colesterol total, LDL, HDL, triglicerídeos, obesidade e proteína C-reativa. A condição de saúde, higiene oral e exame periodontal consistiram nos seguintes parâmetros: presença de saburra, número de bolsas periodontais com profundidade clínica de sondagem $\geq 5 \mathrm{~mm}$; número de sítios com perda de inserção clínica $\geq 6 \mathrm{~mm}$; índice gengival, índice de placa, número de pacientes e sítios com exsudato e perda dentária. Os resultados mostraram que os parâmetros de higiene oral $(p=0.0237)$ e periodontais apresentaram valores significativamente diferentes quando comparados os grupos experimentais $(p<0.0001)$, indicando que pacientes com doença periodontal têm maior probabilidade de ter problemas coronarianos $($ Odds Ratio $=3.913 ; 95 \% \mathrm{IC}=1.326-11.550 ; p=0.0125)$. A conclusão deste estudo sugere que a doença periodontal pode representar um importante papel com repercussão sistêmica e deve ser considerada como possível fator de risco da doença aterosclerótica coronariana justificada pelos parâmetros clínicos avaliados.

Palavras-chave: Periodontite. Aterosclerose Saúde bucal. Doença coronariana. 


\begin{abstract}
Several scientific works showed a significant association between periodontal parameters and cardiovascular problems. The aim of this research went to evaluate to oral health and the periodontal disease in humans verifying if these conditions potentially could be correlated as possible risk factors for coronary atherosclerotic disease. Eighty patients were submitted the coronary angiography and divided in two groups: patients with coronary atherosclerotic disease $(n=59)$ and patients without coronary atherosclerotic disease $(n=21)$. The analyzed variables were age, gender, smoking, alcoholic using, hypertension, diabetes, total cholesterol, LDL, HDL, triglycerides and obesity; reactive C-protein. The periodontal exam and of the condition of health and oral hygiene consisted of the following parameters: tongue biofilm presence, number of periodontal pockets with clinical depth $\geq 5 \mathrm{~mm}$; number of sites with loss of clinical insert $\geq 6 \mathrm{~mm}$; gingival index, plaque index, number of patients and sites with exudates, dental loss. The results showed the parameters of oral hygiene $(p=0.0237)$ and the periodontal condition presented values significantly different when compared the groups $(p<0.0001)$, indicating that patients with periodontal disease have larger probability of having coronary problems (Odds Ratio $=3.913 ; 95 \% \mathrm{IC}=1.326-11.550 ; p=0.0125)$. The conclusion of this study suggests that periodontal disease may to represent an important role with systemic impact and could be considered as a possible risk factor for coronary atherosclerosis, justified by clinical parameters evaluated.
\end{abstract}

Keywords: periodontitis, atherosclerosis, oral health, coronary diseases

\section{Introdução}

Nos últimos anos uma grande quantidade de informações procurou direcionar a atenção para explorar e entender o papel que a doença bucal crônica, precisamente a doença periodontal, poderia representar sobre a saúde sistêmica. Principalmente se associando as doenças coronarianas e suas consequências, como a angina estável, a angina instável e o infarto agudo do miocárdio (CUETO et al., 2005).

A doença periodontal instala-se a partir da colonização bacteriana na cavidade oral. As ações dos patógenos periodontais podem agir diretamente nas células do endotélio vascular ou indiretamente por efeito sistêmico através de suas toxinas. Ambas as ações promovem o início do processo de indução da aterosclerose, induzindo a liberação de mediadores pró inflamatórios pelos monócitos do hospedeiro ou, ainda, podendo levar à formação de trombos agudos em uma pré-existente placa aterosclerótica instável, causando assim eventos tromboembólicos (BECK et al., 1999).

Dessa forma, confirmado o fato de bacteremias poderem ocorrer de forma assintomática, transitória e quotidiana, alguns pesquisadores buscaram um modelo para a relação fisiopatológica, na qual uma pobre saúde oral e periodontal influenciariam nas condições sistêmicas. Observou-se associação positiva entre as endotoxinas produzidas pelos patógenos periodontais (GEERTS et al., 2002) e uma possível elevação dos níveis sorológicos de citocinas por exemplo, a interleucina $1 \beta$, interleucina 6 , proteínas de fase aguda como a proteína $\mathrm{C}$-reativa, além do fibrinogênio provocado pela resposta imunoinflamatória do hospedeiro (DE NARDIN, 2001; LIBBY et al., 2002; PASCERI et al., 2000).

Os fatores de risco conhecidos da doença coronária, tais como a hipertensão arterial, hipercolesterolemia, diabetes e tabagismo não justificam todas as variações nas incidências das doenças 
cardiovasculares. Um terço de todos os infartos do miocárdio ocorre em indivíduos que não apresentam estes fatores e, assim, outros fatores de risco, ainda desconhecidos, podem representar um papel importante (HENNEKENS, 1998), incluindo as infecções crônicas bucais (ABOU-RAYA et al., 2002).

O objetivo da pesquisa foi avaliar e discutir a hipótese atual de que a infecção periodontal é um possível fator de risco para doença coronariana, procurando esclarecer a relação entre o grau de comprometimento dos tecidos periodontais de indivíduos com e sem doença coronariana.

\section{Materiais e métodos}

Um total de 80 indivíduos foi avaliado no setor de emergência do Hospital Bom Jesus, com indicação clínica para cinecoronariografia. O estudo delineou as amostras em dois grupos baseado nos resultados cinecoronariográficos: indivíduos com doença aterosclerótica coronariana e indivíduos sem doença aterosclerótica coronariana.

$\mathrm{O}$ projeto de pesquisa foi realizado com a permissão da Direção Clínica e Comissão de Ética do Hospital Bom Jesus e posteriormente submetido à aprovação do Comitê de Ética da Universidade Estadual de Ponta Grossa, com o parecer $n^{\circ}$ 30/2007 e protocolo 04998/06, ao cumprimento dos princípios éticos contidos na declaração de Helsinki e na legislação específica do país. Todos os participantes da pesquisa assinaram o termo de consentimento livre e esclarecido.

Os critérios de inclusão requeridos foram que toda a população alvo realizasse cinecoronariografia e não apresentasse outras doenças sistêmicas associadas (por exemplo, doença renal, neoplasia), além de não ter realizado nenhum tipo de tratamento periodontal nos seis meses anteriores ao exame odontológico. Os critérios de exclusão adotados eliminaram as pacientes gestantes, crianças, deficientes mentais e aqueles, cujas condições físicas impediriam o exame bucal satisfatório.

O grupo com doença aterosclerótica coronariana consistiu de 59 indivíduos (40 homens e 19 mulheres; média de idade 60-44 anos \pm 9.79) que sofreram anginas ou infarto agudo do miocárdio confirmado pelo exame de cateterismo, o qual constatou a presença de lesão aterosclerótica coronariana.

O grupo sem doença aterosclerótica coronariana foi formado por 21 indivíduos ( 10 homens e 11 mulheres; média de idade de 50-33 anos \pm 9.95) que apresentaram indicação clínica para o cateterismo, porém não foi identificada lesão aterosclerótica coronariana. Posteriormente, cada grupo foi dividido em dois subgrupos: com doença periodontal e sem doença periodontal.

A técnica da cinecoronariografia foi realizada no serviço de hemodinâmica do hospital que consistiu na punção da artéria femoral direita, introduzido um cateter com progressão até o ventrículo esquerdo e cateterizadas seletivamente as artérias coronária direita e esquerda, em várias projeções.

As covariáveis analisadas foram idade, sexo, tabagismo, definido como indivíduo fumante atual, etilismo, hipertensão arterial sistêmica (HAS), definida como pressão arterial igual ou superior a 139/89 $\mathrm{mmHg}$; diabete melito, definida como glicemia de jejum superior a $100 \mathrm{mg} / \mathrm{dl}$, dosagem sérica de colesterol total (valor de referência até 200 $\mathrm{mg} / \mathrm{dl}$ ), lipoproteína de baixa densidade LDL (valor de referência até $130 \mathrm{mg} / \mathrm{dl}$ ), lipoproteína de alta densidade HDL (valor de referência acima de 40 $\mathrm{mg} / \mathrm{dl}$ para homens e acima de 50 para mulheres), triglicerídeos (valor de referência até $150 \mathrm{mg} / \mathrm{dl}$; obesidade, considerado o índice de massa corporal IMC $\geq 25$ (IMC $=$ peso em quilograma dividido pela altura ao quadrado) e proteína C-reativa (PCR). Todos os dados foram coletados pelo pesquisador, anotados em ficha clínica e os prontuários dos pacientes foram consultados. Os dados sobre tabagismo, etilismo foram obtidos através de anamnese.

Os dentes presentes e ausentes das arcadas dentária superior e inferior foram avaliados. Foram examinadas seis faces por dente (mesio-vestibular, vestibular, disto vestibular, mesio-lingual, lingual e disto-lingual), o que possibilitou a sondagem por sítios. As informações sobre a qualidade da higiene oral e razão da perda dentária foram obtidas através de anamnese.

Os parâmetros usados para doença da cavidade oral foram: números de bolsas periodontal com 
profundidade clínica de sondagem $\geq 5 \mathrm{~mm}$ (NBP), número de sítios com perda de inserção clínica (NPIC), índice de sangramento gengival (IG), índice de placa (IP) e números de sítios com exsudato purulento nas bolsas periodontais, além da presença de biofilme no dorso da língua (saburra) e qualidade de higiene e saúde oral. O NPIC foi avaliado somando o valor da recessão gengival mais a profundidade de sondagem de bolsa periodontal. Os indivíduos que apresentaram sítios com NBP, mobilidade dentária, perdas dentárias causadas por periodontites, quadro de inflamação gengival denominado de gengivite e presença ou não de exsudato purulento nos sítios avaliados foram classificados como portadores de doença periodontal. Para as análises estatísticas foram usadas tabelas de contingência e porcentagem. Os dados foram analisados através do teste exato de Fisher, Qui-quadrado, Análise de Variância, teste de Tukey e teste $t$ de Student independente ao nível de significância de 5\%, utilizando os softwares Biostat 4.0, Graph-PAD e Excel 2007.

\section{Resultados}

Dos 80 indivíduos avaliados 59 deles apresentaram cinecoronariografia com lesão acima de $30 \%$ em pelo menos uma artéria coronariana, perfazendo $73,7 \%$ da população avaliada; 21 deles não apresentaram lesões em nenhuma artéria coronária, perfazendo $26,3 \%$ da amostra. Os resultados da cinecoronariografia (Tabela 1) demonstraram que o número de lesões coronarianas acima de $50 \%$ foi maior na artéria descendente anterior $(77,8 \%)$ e circunflexa $(61,1 \%)$ em indivíduos diagnosticados com doença periodontal (DP) $(p<0.0001)$. A Tabela 2 demonstra que os subgrupos não apresentaram diferenças estatísticas quando o critério foram os fatores de risco relatados na literatura.

Os valores médios de glicemia e triglicerídeos dos indivíduos com doença aterosclerótica coronariana e com doença periodontal apresentaram-se maiores e acima do valor de referência, porém não demonstraram valores estatísticos significativamente diferentes, embora indicassem uma tendência à associação entre disfunção endócrina, triglicerídeos e doença periodontal nos pacientes portadores de doença aterosclerótica. Comparando o subgrupo com doença aterosclerótica coronariana e com doença periodontal versus o subgrupo sem doença aterosclerótica coronariana e sem doença periodontal, os valores dos níveis de colesterol apresentaram-se mais elevados para os indivíduos com doença aterosclerótica coronariana e com doença periodontal. Esses valores apresentaram-se estatisticamente diferentes $(p=0.0389)$ segundo teste $\mathrm{t}$ de Student, conforme demonstrado na Tabela 3 .

Os resultados da Tabela 4 mostraram que a associação da doença periodontal com a doença aterosclerótica coronariana ficou evidentes em quantidade de números de bolsas periodontal com profundidade clínica de sondagem $\geq 5 \mathrm{~mm}(p<0.0001)$, números de sítios com perda de inserção clínica $\geq$ $6 \mathrm{~mm}(p<0.0001)$, números de sítios com exsudato purulento das bolsas periodontais $(p<0.0001)$, qualidade de higiene e saúde oral $(p=0.0237)$ e maior perda dentária por periodontite $(p<0.0001)$. $\mathrm{O}$ índice gengival apresentou uma tendência para um valor mais elevado nos pacientes com doença aterosclerótica, demonstrando maior inflamação gengival $(p=0.0072)$. Na análise estatística de Anova-Tukey, o IG foi estatisticamente significante quando comparados os subgrupos com doença aterosclerótica coronariana e com doença periodontal versus o grupo sem doença aterosclerótica e sem doença periodontal $(p<0.05)$, e versus o subgrupo de pacientes com doença aterosclerótica coronariana e sem doença periodontal $(p<0.05)$.

A distribuição da frequência dos 80 indivíduos foi tabulada e apresentada na Tabela 5. O teste exato de Fisher demonstrou um resultado altamente significativo $(p=0.0125)$. A diferença entre as proporções foi de 0.2519 ou $25,19 \%$, sendo que $95 \%$ do intervalo de confiança da diferença foi estimado entre 0.05876 e 0.4450 . O valor de Odds Ratio foi de 3.913 (95\% do intervalo de confiança $=1.326-11.550$ ), indicando que um indivíduo com doença periodontal tem aproximadamente quatro vezes maior probabilidade de desenvolver doença aterosclerótica coronariana. 
Tabela 1 - Dados estatísticos em porcentagem e teste Qui-quadrado da coronariografia nos indivíduos com/ sem doença aterosclerótica coronariana (DAC), mostrando lesões entre $30 \%$ e $50 \%$ e acima de $50 \%$ divididos em indivíduos com/sem doença periodontal (DP).

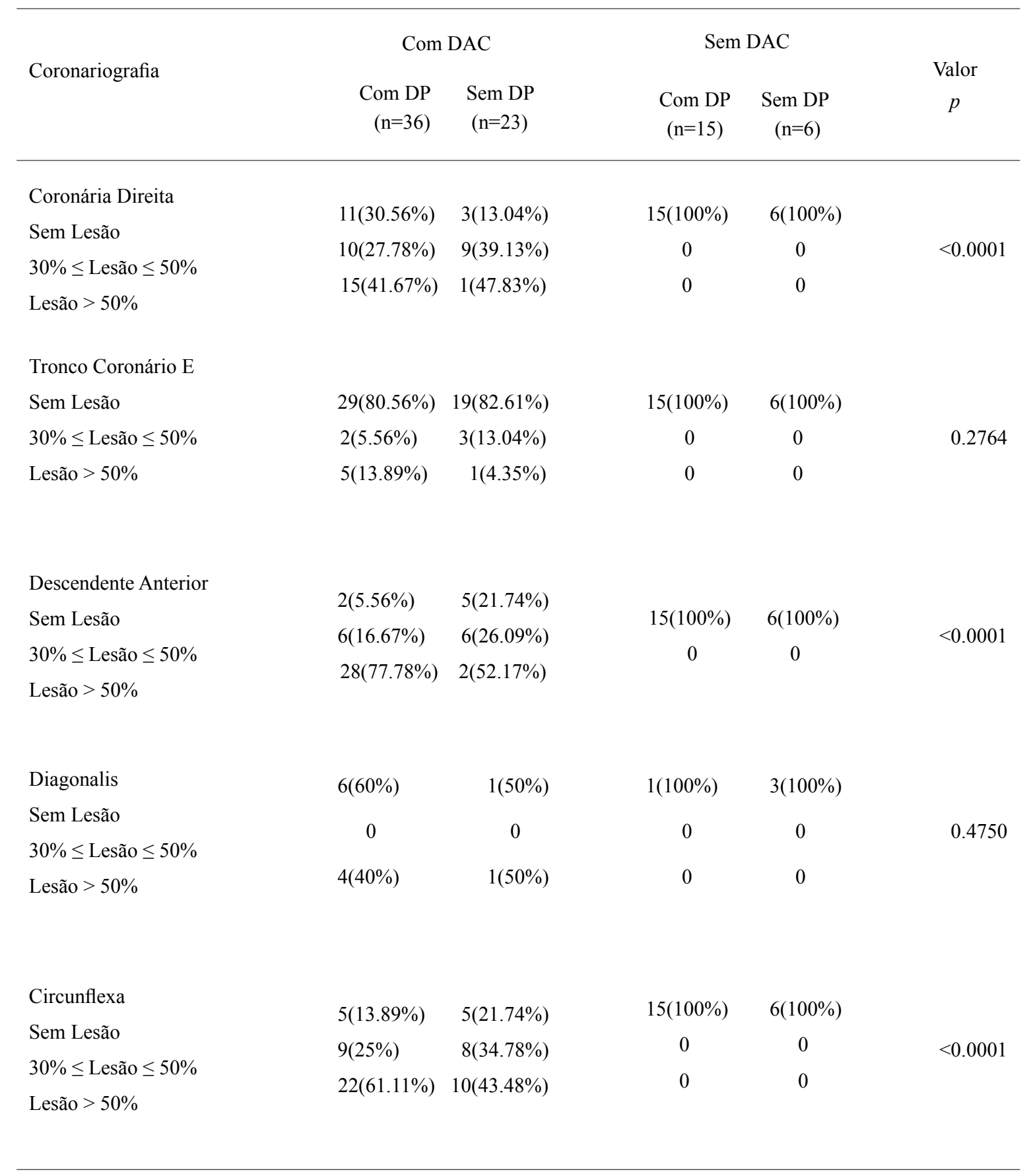


Tabela 2 - Dados biodemográficos e de fatores de risco em porcentagem, teste Anova e Qui-quadrado nos indivíduos com/sem doença ateroclerótica coronariana associada ou não com doença periodontal.

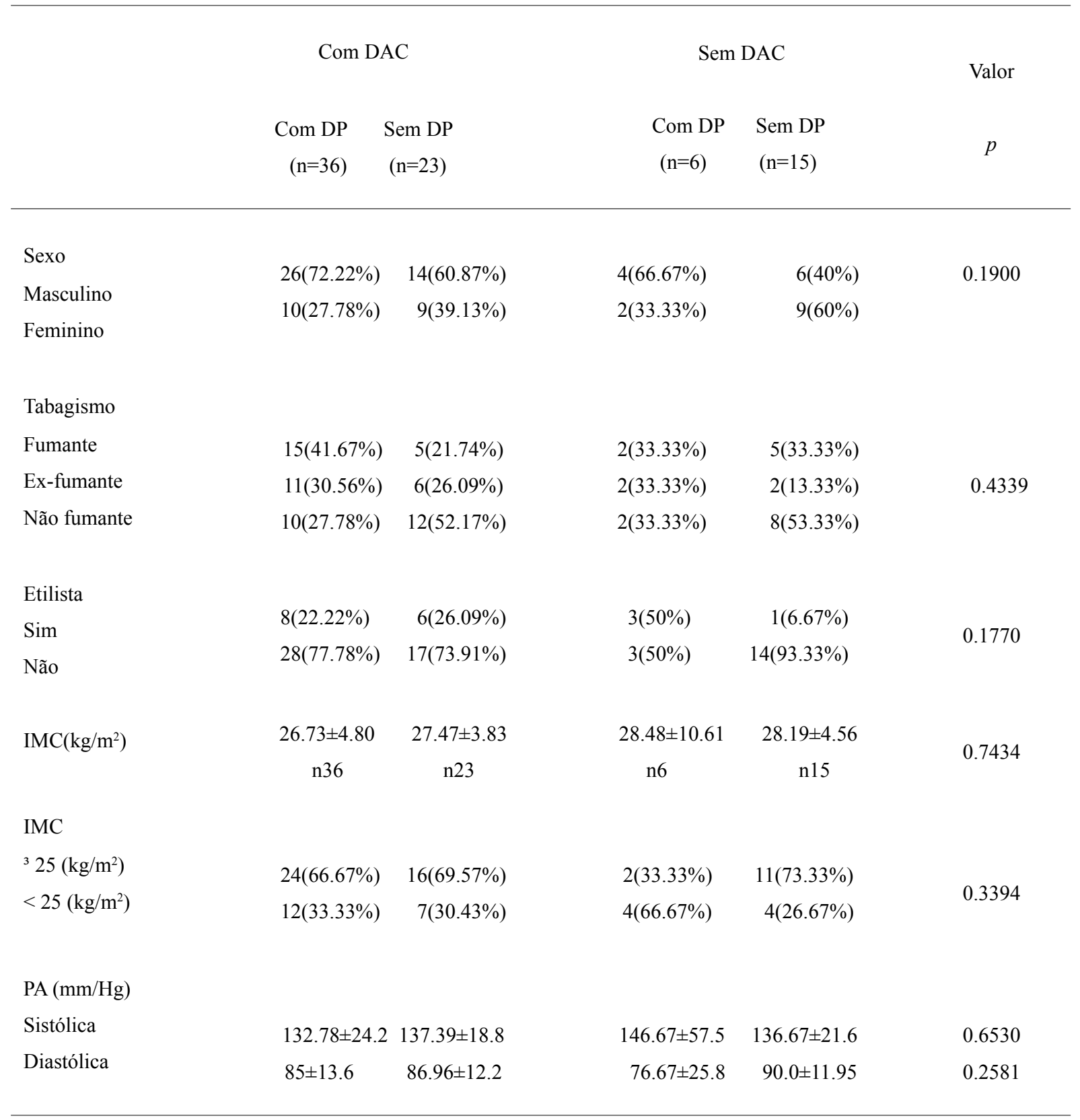

Nota: $(*) p=0.0389$ versus pacientes sem doença aterosclerótica e sem doença periodontal (teste t de Student) 
Tabela 3 - Dados estatísticos em teste Anova de exames sanguíneos nos indivíduos com/sem doença aterosclerótica coronariana associada ou não a doença periodontal.

\begin{tabular}{|c|c|c|c|c|c|}
\hline & \multicolumn{2}{|r|}{ Com DAC } & \multicolumn{2}{|r|}{ Sem DAC } & \multirow{2}{*}{$\begin{array}{c}\text { Valor } \\
p\end{array}$} \\
\hline & $\begin{array}{r}\text { Com D } \\
(\mathrm{n}=36\end{array}$ & $\begin{array}{l}\text { Sem DP } \\
(\mathrm{n}=23)\end{array}$ & $\begin{array}{r}\text { Com DP } \\
(\mathrm{n}=6)\end{array}$ & $\begin{array}{l}\text { Sem DP } \\
(\mathrm{n}=15)\end{array}$ & \\
\hline $\begin{array}{l}\text { Glicemia } \\
(\mathrm{mg} / \mathrm{dl})\end{array}$ & \multicolumn{2}{|c|}{$132.06 \pm 59.66 \quad 127.65 \pm 49.2$} & $114.17 \pm 6.82$ & $111.33 \pm 23.66$ & 0.5309 \\
\hline $\begin{array}{l}\text { Colesterol Total } \\
(\mathrm{mg} / \mathrm{dl})\end{array}$ & $181.2 \pm 44.2^{*}$ & $165.74 \pm 42.2$ & $184.8 \pm 62.6$ & $155.5 \pm 22.9$ & 0.1756 \\
\hline $\begin{array}{l}\mathrm{HDL} \\
(\mathrm{mg} / \mathrm{dl})\end{array}$ & $44.03 \pm 14.70$ & $53.43 \pm 29.37$ & $44.00 \pm 5.97$ & $41.53 \pm 11.29$ & 0.2122 \\
\hline $\begin{array}{l}\mathrm{LDL} \\
(\mathrm{mg} / \mathrm{dl})\end{array}$ & $93.53 \pm 28.53$ & $88.00 \pm 31.95$ & $107.17 \pm 49.37$ & $7 \quad 87.00 \pm 16.75$ & 0.4804 \\
\hline $\begin{array}{l}\text { Triglicerídeos } \\
(\mathrm{mg} / \mathrm{dl})\end{array}$ & $186.2 \pm 124.3$ & $160.0 \pm 49.4$ & $165.8 \pm 76.0$ & $155.8 \pm 50.8$ & 0.6385 \\
\hline $\begin{array}{l}\text { PCR } \\
(\mathrm{mg} / \mathrm{dl})\end{array}$ & $\begin{array}{l}0.66 \pm 1.20 \\
(n=32)\end{array}$ & $\begin{array}{c}0.88 \pm 1.73 \\
(n=15)\end{array}$ & $\begin{array}{l}0.24 \pm 0.33 \\
(n=5)\end{array}$ & $\begin{array}{l}0.60 \pm 1.59 \\
(n=9)\end{array}$ & 0.8328 \\
\hline PCR & $4(12.5 \%)$ & $1(6.67 \%)$ & \multirow{3}{*}{$\begin{array}{l}2(40 \%) \\
3(60 \%)\end{array}$} & \multirow{3}{*}{$\begin{array}{l}1(12.5 \%) \\
8(87.5 \%)\end{array}$} & \multirow{3}{*}{0.2884} \\
\hline Reagente & $28(87.5 \%)$ & $14(93.33 \%)$ & & & \\
\hline Não Reagente & & & & & \\
\hline
\end{tabular}

Nota: $(*) p=0.0389$ versus pacientes sem doença aterosclerótica e sem doença periodontal (teste t de Student) 
Tabela 4 - Dados estatísticos (porcentagem, Anova, teste de Tukey e Qui-quadrado) da avaliação bucal nos pacientes com/sem doença aterosclerótica coronariana associada ou não a doença periodontal.

\begin{tabular}{|c|c|c|c|c|c|}
\hline & \multicolumn{2}{|c|}{ Com DAC } & \multicolumn{2}{|r|}{ Sem DAC } & \multirow{2}{*}{$\begin{array}{l}\text { Valor } \\
\text { p }\end{array}$} \\
\hline & $\begin{array}{r}\text { Com DP } \\
(n=36)\end{array}$ & $\begin{array}{l}\text { Sem DP } \\
(\mathrm{n}=23)\end{array}$ & \multicolumn{2}{|c|}{$\begin{array}{r}\text { Com DP } \\
\qquad(n=6)\end{array}$} & \\
\hline $\begin{array}{l}\text { Dentes (Faces) } \\
\text { Presentes }\end{array}$ & $325(1950)$ & $56(336)$ & \multirow{2}{*}{\multicolumn{2}{|c|}{$\begin{array}{l}115(690) \\
305(1830)\end{array}$}} & \multirow{2}{*}{$<0.0001$} \\
\hline Ausentes & $683(4098)$ & $588(3528)$ & & & \\
\hline IG & $1.34 \pm 0.50^{*}$ & $0.60 \pm 0.43$ & $1.31 \pm 0.53$ & $0.81 \pm 0.49$ & 0.0072 \\
\hline IP & $2.51 \pm 0.93$ & $1.79 \pm 0.34$ & $2.79 \pm 0.67$ & $1.95 \pm 0.73$ & 0.1127 \\
\hline $\begin{array}{l}\mathrm{NPB} \\
{ }^{3} 5 \mathrm{~mm}\end{array}$ & $442(22.67 \%)$ & 0 & \multirow{2}{*}{\multicolumn{2}{|c|}{$\begin{array}{lr}164(35.04 \%) & 0 \\
304(64.96 \%) & 690(100 \%)\end{array}$}} & \multirow{2}{*}{$<0.0001$} \\
\hline$<5 \mathrm{~mm}$ & $1508(77.33 \%)$ & $336(100 \%)$ & & & \\
\hline $\begin{array}{l}\text { NPIC } \\
{ }^{3} 6 \mathrm{~mm}\end{array}$ & $426(21.85 \%)$ & 0 & \multirow{2}{*}{\multicolumn{2}{|c|}{$\begin{array}{l}168(35.90 \%) \quad 21(3.04 \%) \\
300(64.10 \%) 669(96.96 \%)\end{array}$}} & \multirow{2}{*}{$<0.0001$} \\
\hline$<6 \mathrm{~mm}$ & $1524(78.15 \%)$ & $336(100 \%)$ & & & \\
\hline \multicolumn{6}{|l|}{$\begin{array}{l}\text { Pacientes com } \\
\text { Exudato }\end{array}$} \\
\hline Positivo & $24(66.67 \%)$ & $23(100 \%)$ & $2(33.33 \%)$ & $15(100 \%)$ & $<0.0001$ \\
\hline \multicolumn{6}{|l|}{ Negativo } \\
\hline \multicolumn{6}{|l|}{ Sítios com Exudato } \\
\hline Positivo & $\begin{array}{l}72(3.69 \%) \\
1878(96.31 \%)\end{array}$ & $\begin{array}{r}0 \\
336(100 \%)\end{array}$ & $\begin{array}{l}24(5.13 \%) \\
444(94.87 \%)\end{array}$ & $\begin{array}{r}0 \\
690(100 \%)\end{array}$ & $<0.0001$ \\
\hline \multicolumn{6}{|l|}{ Negativo } \\
\hline $\begin{array}{l}\text { Pacientes com Saúde } \\
\text { Higiene Oral } \\
\text { Boa }\end{array}$ & $\begin{array}{l}10(27.78 \%) \\
26(72.22 \%)\end{array}$ & $\begin{array}{r}14(60.87 \%) \\
9(39.13 \%)\end{array}$ & $\begin{array}{l}3(50 \%) \\
3(50 \%)\end{array}$ & $\begin{array}{r}10(66.67 \%) \\
5(33.33 \%)\end{array}$ & 0.0237 \\
\hline \multicolumn{6}{|l|}{ Ruim } \\
\hline $\begin{array}{l}\text { Portadores de Saburra } \\
\text { Positiva } \\
\text { Negativa }\end{array}$ & $\begin{array}{l}18(50 \%) \\
18(50 \%)\end{array}$ & $\begin{array}{r}9(39.13 \%) \\
14(60.87 \%)\end{array}$ & $\begin{array}{l}1(16.67 \%) \\
5(83.33 \%)\end{array}$ & $\begin{array}{r}3(20 \%) \\
12(80 \%)\end{array}$ & 0.1463 \\
\hline
\end{tabular}

Nota: $(*) p<0.05$ versus pacientes com doença aterosclerótica e sem doença periodontal. $p<0.05$ versus pacientes sem doença aterosclerótica e sem doença periodontal 
Tabela 5 - Distribuição da frequência de indivíduos com/sem doença aterosclerótica coronariana versus pacientes com/sem doença periodontal, baseado na na porcentagem, teste exato de Fisher e Odds Ratio (*).

\begin{tabular}{llcc}
\hline & \multicolumn{2}{c}{ Com DAC } \\
& $(\mathrm{n}=59)$ & $\begin{array}{l}\text { Sem DAC } \\
(\mathrm{n}=21)\end{array}$ & $p$ \\
\hline $\begin{array}{l}\text { Com Doença } \\
\text { Periodontal }\end{array}$ & $36(85.71 \%)$ & $6(14.29 \%)$ & 0.0125 \\
Sem Doença & $23(60.53 \%)$ & & \\
Periodontal & & $15(39.47 \%)$ & \\
\hline
\end{tabular}

Nota: (*) Odds Ratio $=3.913$ (95\% IC: $1.326-11.550)$

\section{Discussão}

Muitas pesquisas realizadas com o intuito de avaliar a doença periodontal como fator de risco para doença aterosclerótica não esclareceram os quadros de obstrução coronarianas obtidas através de cinecoronariografia (TUOMINEN et al., 2003; BUHLIN et al., 2005; YLOSTALO et al., 2006; KATZ et al., 2001; CUETO et al. 2005). Nos sinais e sintomas clínicos da doença, há situações em que se faz necessário conhecer o padrão arterial coronário (SOLIMENE et al., 2003). Portanto, a cinecoronariografia é um direcionador que esclarece o diagnóstico do evento coronariano ou estratificação de risco o qual se pode avaliar a presença ou não de coronariopatia obstrutiva de grau importante, tanto em pacientes clínicos como em casos clínicos falsos positivos. $\mathrm{Na}$ indicação clínica para realização da cinecoronariografia, $73,7 \%$ dos pacientes foram diagnosticados com lesões ateroscleróticas acima de $30 \%$ e $26,3 \%$ não apresentaram lesões ateroscleróticas coronarianas.

As cinecoronariografias dos indivíduos agrupados mostraram que as artérias descendentes anteriores e circunflexas em indivíduos com aterosclerose coronariana e lesões acima de 50\% apresentaram maior número de indivíduos com doença periodontal e suas sequelas, evidenciando que nos casos de obstrução severa, a doença periodontal estava presente e tinha maior associação.

As bactérias são os principais causadores da doença periodontal, colonizando os sítios subgen- givais e organizando-se através de um biofilme dentário que é resistente às respostas do hospedeiro e muitas vezes, ao uso local ou sistêmico de antimicrobianos, sendo potencialmente capazes de invadir os tecidos periodontais (PAGE et al., 1998).

A aterosclerose se desenvolve como resposta a uma injúria que é primariamente um processo inflamatório crônico, na qual uma infecção pode atuar como mais um fator de risco para perpetuar a inflamação. O processo envolvido está estritamente relacionado a mecanismos normais de proteção associados à inflamação e reparo, mas o dano pertinente resulta numa resposta crônica no endotélio arterial (ROSS, 1999). Essa doença tem caráter evolutivo, podendo progredir até a oclusão da luz da artéria coronária e risco de instabilização das placas ateroscleróticas, levando a eventos agudos como angina instável e o infarto agudo do miocárdio (STARY et al., 1995; RAMIRES et al., 2003; PAQUETE et al., 2007). Relacionados a essas ocorrências agudas, temos também níveis aumentados de proteína de fase aguda como fator necrose tumoral (TNF- $\alpha$ ), interlucinas (PASCERI et al., 2000), proteína C-reativa (LIBBY et al., 2002; SANTOS et al., 2003) e níveis séricos enzimáticos de CK e CKMB (HUGGON et al., 2001).

A coexistência da doença periodontal como fator de risco para doença aterosclerótica coronariana e suas manifestações clínicas parece bem interessante. Os resultados obtidos demonstraram que a associação entre doença periodontal e eventos ateroscleróticos coronarianos foram bastante 
significativos estando de acordo com os resultados de Matilla et al. (1989). Na avaliação dos nossos dados, quando os indivíduos com ou sem doença aterosclerótica coronária foram agrupados com os indivíduos com ou sem doença periodontal, a presença de doença aterosclerótica coronariana teve associação significativa com a doença periodontal. Ao analisar os parâmetros da cavidade oral os resultados mostraram ser estatisticamente diferentes $(p<0.0001)$ explicando associação entre as doenças. Procurando direcionar o raciocínio ao número de perdas dentárias, foi numericamente maior nas associações da doença aterosclerótica coronariana e na doença periodontal, explicando o fato de que a doença periodontal é a maior responsável pela perda dos dentes em adultos.

A justificativa que envolve a doença periodontal e a doença aterosclerótica coronariana se dá na possibilidade de bactérias patogênicas disseminarem para corrente sanguínea através de constante bacteremia (DALY et al., 2001; IWAI et al 2009) e assim induzir uma resposta inflamatória crônica nos vasos coronarianos (PAQUETE et al., 2007). Evidências científicas apontaram a ação da Porphyromonas gingivalis na capacidade de estimular a formação de células espumosas pela agregação do LDL (MIYAKAWA et al., 2004) e influenciar no metabolismo lipídico (RUFAIL et al., 2007). Outros patógenos periodontais têm sido associados às placas ateroscleróticas como Agregatibacter actinomycetemcomitans, Tannerella forsythia e Prevotella intermedia (PADILLA et al., 2006; TAYLOR-ROBINSON et al., 2002), demonstrando que as doenças periodontais são prevalentes nos pacientes com cardiopatias isquêmicas quando avaliadas em conjunto.

Considera-se que tanto a doença coronariana quanto a doença periodontal são doenças multifatoriais e que nas análises dos resultados obtidos precisam ser levados em consideração os fatores de riscos dos grupos (BARILLI et al., 2006; MONTEIRO et al. 2009). Atenção especial foi dada aos subgrupos, tentando-se minimizar as variáveis entre eles, ou seja, os vários fatores de risco que poderiam influenciar nos resultados, demonstrando que muitas variáveis não foram determinantes uma vez que não foram observadas diferenças estatísticas.
No que se refere ao colesterol total os valores dos níveis apresentaram-se mais elevados para os pacientes com doença aterosclerótica coronariana e com doença periodontal, sendo esses valores estatisticamente significantes $(p=0.0389)$, indicando que pode também existir uma possível ligação entre inflamação periodontal crônica, aterosclerose e hipercolesterolemia (RUFAIL et al., 2007; KATZ et al., 2001; YLOSTALO et al., 2010). Além disso, níveis de glicose também podem ser relevantes em pacientes que apresentam diabetes melito (NIBALI et al., 2007, JANKET et al, 2008) especialmente porque os valores glicêmicos tendem a ser superiores no grupo de indivíduos com doença aterosclerótica e com doença periodontal.

Outras pesquisas mostraram que a doença periodontal é uma infecção que pode aumentar a produção de marcadores inflamatórios e fatores de coagulação sanguínea como a proteína C-reativa (JOSHIPURA et al. 2004; BUHLIN et al., 2009) e o fibrinogênio (DE NARDIN, 2001; BUHLIN et al., 2009). Quando a doença periodontal é tratada ocorre uma redução significante nos níveis da proteína C-reativa após o tratamento não cirúrgico de periodontite avançada em indivíduos sistemicamente saudáveis (D'AIUTO et al., 2006). Embora existam evidências que apontem um aumento dos níveis séricos de proteína C-reativa em indivíduos com periodontite crônica, investigações são necessárias para suportar o tratamento periodontal como principal controladora da inflamação sistêmica na população em geral (IOANNIDOU et al., 2006). Em nosso estudo, a proteína C-reativa não apresentou uma diferença significativa que pudesse associar os seus níveis com o quadro de inflamação sistêmica da aterosclerose com a doença periodontal.

Os dados estatísticos demonstraram que existe uma Odds Ratio de 3.913 (IC 95\%: 1.326 a 11.550) entre as doenças, indicando que um indivíduo com doença periodontal tem quatro vezes maior chance de ter problemas coronarianos, especialmente porque os indivíduos com doença aterosclerótica coronariana apresentaram os piores quadros de saúde oral quando comparados com outros subgrupos. Outros estudos também indicam um risco aumentado do desenvolvimento de doença 
aterosclerótica coronariana e infarto agudo do miocárdio entre os indivíduos com periodontite (DELIARGYRIS et al., 2004; ACCARINI et al., 2006; RECH et al., 2007), sugerindo fortemente que a doença periodontal poderia apresentar uma associação com as coronariopatias.

No entanto, os estudos conduzidos não comprovam a causa da associação entre doença periodontal e doença cardiovascular aterosclerótica e suas sequelas (BECK et al., 2005). Portanto, através dos resultados obtidos, consideramos a doença periodontal como um possível fator de risco para a arteriosclerose coronariana, salientando que outras investigações deverão ser realizadas para melhor compreender a relação entre elas. Com isso acreditamos que devido a importância das doenças ateroscleróticas coronarianas sobre a taxa de morbidade e mortalidade (RAMIRES et al., 2003), bem como a relevante prevalência das doenças periodontais e seus efeitos, reforçam a necessidade da realização de mais pesquisas na área, indicando que a doença periodontal representa um exemplo de infecção crônica que pode fornecer uma forte associação para o estudo da relação entre infecções crônicas e a aterogênese humana.

\section{Conclusão}

Concluímos que a doença periodontal representa um importante papel como infecção crônica, com repercussão sistêmica e consideramos também a doença periodontal como possível fator de risco para doença aterosclerótica coronariana, o que se justifica pelos parâmetros clínicos periodontais associadas às lesões coronarianas observadas na população avaliada.

\section{REFERÊNCIAS}

ABOU-RAYA, S. et al. Coronary artery disease and periodontol disease: is there a link? Angiology., v.53, n.2, p.141-48, 2002.

ACCARINI, R.; GODOY, M. F. Doença periodontal como potencial fator de risco para síndromes coronarianas agudas. Arquivo Brasileiro Cardiologia. v.87, p.592-596, 2006.
BARILLI, A. L. A. et al. Doenças periodontais em pacientes com doenças isquêmica coronariana aterosclerótica, em hospital universitário. Arquivo Brasileiro de Cardiologia. v.87, n.6, p.695-700, 2006.

BECK, J. D. et al. Dental infections and atherosclerosis. Am. Heart J., v.138, p.S528-S533, 1999.

BECK, J.D. et al. Systemic effects of periodontitis: epidemiology of periodontal disease and cardiovascular disease. J. Periodontol., v.76, p.2089-2100, 2005.

BUHLIN, K. et al. Oral health in women with coronary heart disease. J. Periodontol. v.76, p.544-550, 2005.

BUHLIN, K. et al. Risk factors for atherosclerosis in case with severe periodontitis. J. Clin. Periodontol., v.36, p.54149, 2009.

CUETO, A. et al. Periodontitis as risk factor for acute myocardial infarction. A case control study of Spanish adults. J. Periodont. Res. v.40, p. 36-42, 2005

D'AIUTO, F. et al. Periodontal infections cause in tradicional and novel cardiovascular risk factors: results from a randomized controlled clinical trial. Am. Heart J., v.151, p. 977-84, 2006.

DALY, C. G. et al. Bacteremia due to periodontal probing: a clinical and microbiological investigation. J. Periodontol., v.72, p.210-214, 2001.

DELIARGYRIS, E. N. et al. Periodontal disease in patients with acute myocardial infarction: prevalence and contribution to elevated c-reactive protein levels. Am. Heart J., v.147, n.6, p.1005-09, 2004.

DE NARDIN, E. The role of inflammatory and immunological mediadors in periodontitis and cardiovascular disease. Ann. Periodontol., v.6, n.1, p.30-40, 2001.

GEERTS, S. O. et al. Systemic release of endotoxins induced by gentle mastication: association with periodontitis severity. J. Periodontol., v.73, p.73-78, 2002.

HENNEKENS, C. H. Increasing burden of cardiovascular disease. Current knowledge and future directions for research on risk factors. Circulation, v.67, n.10, p.1095-102, 1998.

HUGGON. A. M. et al. Biochemical markers in the management of suspected acute myocardial infartion in the emergency department. Emerg. Med. J., v.18, p.15-19, 2001.

IOANNIDOU, E. et al. Effect of periodontal treatment on serum c reactive protein levels: a systematic review and metaanalysis. J. Periodontal., v.77, p.1635-1642, 2006.

IWAI, T. Periodontal bacteremia and various vascular diseases. J. Periodontol. Res, v.44, p.689-94, 2009.

JANKET, S. J. et al. Oral infection, hyperglycemia, and endothelial dysfunction. Oral Surg. Oral Med. Oral Pathol. Oral Radiol. Endod., v.105, p.173-9, 2008. 
JOSHIPURA, K. J. et al. Periodontal disease and biomarkers related to cardiovascular disease. J. Dent. Res., v.83, p.1515, 2004.

KATZ, J. et al. On the association between hypercholesterolemia, cardiovascular disease and severe periodontal disease. J. Clin. Periodontol., v.28, p.865-868, 2001.

LIBBY, P. et al. Inflamation and atherosclerosis. Circulation, v.105, n.9, p.1135-43, 2002.

MATILLA, K. J. et al. Association between dental health and myocardial infarction. BMJ, v.298, n.6676, p.779-81, 1989.

MIYAKAWA, $H$. et al. Interaction of $P$ gingivalis with lowdensity lipoprotein: implications for a role for periodontitis in atherosclerosis. J. Periodontol Res., v.39, p.1-9, 2004.

MONTEIRO, A. M., et al. Cardiovascular disease parameters in periodontitis. J. Periodontol., v.80, n.3, p.378-88, 2009.

NIBALI, L. et al. Severe periodontitis is associated with systemic inflammation and a dysmetabolic status: a casecontrol study. J. Clin. Periodontol. v.34, p. 931-937, 2007.

PADILLA, C. et al. Periodontal pathogens in atheromatous plaques isolated from patients with chronic periodontitis. $\mathbf{J}$. Periodont. Res., v.41, p.350-353, 2006.

PAGE, R. C, et al. The pathobiology of periodontal disease may affect systemic disease: inversion of a paradigm. Ann. Periodontol., v.3, p.108-120, 1998.

PAQUETE, D. W. et al. Cardiovascular desease, inflamation, and periodontal infection. Periodontology 2000, v.44, p.113126, 2007.

PASCERI, V. et al. Direct proinflammatory effect of c-reative protein on human endothelial cells. Circulation, v.102, p.2165-8, 2000.

RAMIRES, J.A.F. et al. Insuficiência coronariana crônica. Rev Bras Med., v.60, n.7, p.415-28, 2003.

RECH, L. R. et al. Associação entre doença periodontal e síndrome coronariana aguda. Arquivo Brasileiro de Cardiologia, v.88, n.2, p.185-190, 2007.

ROSS, R. Atherosclerosis: an inflammatory disease. N. Engl. J. Med., v.340, p.115-126, 1999.

RUFAIL, M. L. et al. Atherogenic lipoprotein parameters in patients with aggressive periodontitis. J. Periodont. Res., v.42, p.495-502, 2007.

SANTOS, W.B. et al. Proteína c reativa e doença cardiovascular. As bases da evidência científica. Arquivo Brasileiro de Cardiologia, v.80, n.4, p.452-6, 2003.

SOLIMENE, M. C. et al. Indicações de cinecoronariografia na doença arterial coronária. Rev. Assoc. Med. Bras., v. 49, n. 2, p.203-9, 2003.

STARY, H.C. et al. A definition of advanced types of atherosclerotic lesions and a histological classification of atherosclerosis. Circulation, v.92, n.5, p.1355-74, 1995.

TAYLOR-ROBINSON, D.et al. Oro-dental bacteria in various atherosclerotic arteries. Eur. J. Clin. Microbiol. Infect Dis., v.21, p.755-757, 2002.

TUOMINEN, R. et al. Oral health indicators poorly predict coronary heart disease death. J. Dent Res., v.82, n.9, p.7138, 2003.

YLOSTALO, P., et al. Gingivitis, dental caries and tooth loss: risk factors for cardiovascular disease or indicators of elevated health risks. J. Clin. Periodontol., v.33, p.92-101, 2006.

YLOSTALO, P., et al. Periodontal infection and subclinical atherosclerosis: the role of high-density lipoprotein as a modifying factor. J. Clin. Periodontol., v.37, p.617-24, 2010. 